\title{
SOLVING HIGHER ORDER FUCHS TYPE DIFFERENTIAL SYSTEMS AVOIDING THE INCREASE OF THE PROBLEM DIMENSION
}

\author{
E. NAVARRO, L. JÓDAR and R. COMPANY \\ Departamento de Matemática Aplicada \\ Universidad Politécnica de Valencia \\ P.O. Box 22.012, Valencia, Spain
}

(Received June 26,1992$)$

\begin{abstract}
In this paper, we develop a Frobenius matrix method for solving higher order systems of differential equations of the Fuchs type. Generalized power series solution of the problem are constructed without increasing the problem dimension. Solving appropriate algebraic matrix equations a closed form expression for the matrix coefficient of the series are found. By means of the concept of a $k$-fundamental set of solutions of the homogeneous problem an explicit solution of initial value problems are given.
\end{abstract}

KEY WORDS AND PHRASES. Fuchs type system, series solution, algebraic matrix equation, co-solution, $k$-fundamental set.

1991 AMS SUBJECT CLASSIFICATION CODES. 34A25, 15A24.

\section{INTRODUCTION.}

Numerous problems from chemistry, physics and mechanics are related to systems of differential equations of the type, ([10], [9]),

$$
t^{n} x^{(n)}+t^{n-1} P_{n-1}(t) x^{(n-1)}+\cdots+P_{o}(t) x=0,
$$

where the unknown $x(t)$ is a $C_{m x 1}$ valued function and $P_{j}(t)$ is a $C_{m x m}$ values analytic matrix function in the interval $|t|<a$. Standard techniques are based on the consideration of the change $w_{1}=x, t w_{1}^{\prime}=w_{2}, \cdots, t w_{n-1}^{\prime}=w_{n}$, and the extended system

$$
t\left[\begin{array}{c}
w_{1}^{\prime} \\
\vdots \\
w_{n}^{\prime}
\end{array}\right]=\left[\begin{array}{c|ccccc}
0 & I & 0 & \cdots & & 0 \\
\vdots & \vdots & I & I & \ddots & \vdots \\
0 & 0 & & I \\
\hdashline A_{o}(t) & A_{1}(t) & & \cdots & & A_{n-1}(t)
\end{array}\right]\left[\begin{array}{c}
w_{1} \\
\vdots \\
w_{n}
\end{array}\right]
$$

where $A_{j}(t)$ for $0 \leqq j \leqq n-1$, is a linear combination of $P_{j}(t)$ with constant coefficients and are therefore analytic in $|t|<a$, see $([1],[4]$ and [5]), for details.

The consideration of system (1.2) to study problems related to (1.1) or non-homogeneous problems of the type

$$
t^{n} x^{(n)}+t^{n-1} P_{n-1}(t)+\cdots+P_{0}(t) x=g(t), t \varepsilon J, g(t) \varepsilon C_{m x 1}
$$

has the computational drawback of the increase of the problem dimension apart from the lack of explicitness of the series solution due to the relationship $x(t)=[I, 0, \cdots, 0] W(t)$, where $W(t)=\left(w_{1}(t), \cdots, w_{n}(t)\right)^{T}$. Note that as it happens in the scalar case, the Frobenius approach does 
not provide explicit series solution for the corresponding problem (1.1) when one considers the equivalent extended system (1.2). The aim of the paper is to construct explicit series solutions for (1.1) and to obtain a closed form solution of initial value problems for the non-homogeneous system (1.3) without increasing the problem dimension.

The organization of the paper is as follows. In section 2 we solve in a closed form way algebraic matrix equations of the type

$$
X P^{n}+T_{n-1} X P^{n-1}+T_{n-2} X P^{n-2}+\cdots+T_{1} X P+T_{0} X=S,
$$

where $P \varepsilon \mathrm{C}_{p x p}, S \varepsilon \mathrm{C}_{m x p}, T_{i} \varepsilon \mathrm{C}_{m x m}$ or $0 \leq i \leq n-1$, and the unknown $X$ lies in $\mathrm{C}_{m x p}$. Such equations appear in the construction of matrix series solutions for system (1.1) without increasing the problem dimension. Following the ideas developed in [6] for time invariant equations, we introduce in section 3 the concept of a $k$-fundamental set of solutions for systems of the type

$$
y^{(n)}+Q_{n-1}(t) y^{(n-1)}+\cdots+Q_{0}(t) y=0,
$$

where $Q_{j}(t) \varepsilon C_{m x m}$ is a continuous function on an interval $J$, for $0 \leq j \leq n-1$. Section 4 deals with the construction of matrix powers series solution of (1.1) as well as the proof of its convergence and the construction of $k$-fundamental sets of solutions of (1.1) composed by generalized power series matrix solutions of (1.1). Then a closed form solution of the general solution of initial value problems for system (1.3) and without increasing the problem dimension is given.

If $A$ is a matrix in $C_{m x n}$ and $B^{H}$ denotes the conjugate transpose of $B$, we denote by $\|B\|$ its spectral norm, defined by the maximum eigenvalue of the set $\left\{|z|^{\frac{1}{2}} ; z\right.$ is an eigenvalue of $B^{H} B$.

\section{ALGEBRAIC RESULTS.}

We begin this section with a closed form solution for the algebraic matrix equation (1.4).

THEOREM 1. Let $P \varepsilon \mathrm{C}_{p x p}, S \varepsilon \mathrm{C}_{m x p}, T_{i} \varepsilon \mathrm{C}_{m x m}$ for $0 \leq i \leq n-1$, and let $H$ be the matrix

$$
H=\left[\begin{array}{c|ccc}
0 & I & \ddots \\
\vdots & & \ddots & I \\
0 & \mid &
\end{array}\right]
$$

such that

$$
\sigma(H) \cap \sigma(P)=\emptyset
$$

Then equation (1.4) has only one solution. If $q(z)=\sum_{j=0}^{r} a_{j} z^{j}$ is an annihilating polynomial of $\boldsymbol{H}, \boldsymbol{M}=\left(\boldsymbol{M}_{i j}\right)$ is an invertible matrix in $\mathrm{C}_{m n x m n}$ with $\boldsymbol{M}_{i j} \varepsilon C_{m x n_{j}}, 1 \leq i \leq n, 1 \leq j \leq k$, and $W=\left(W_{s i}\right)=M^{-1}, W_{s i} \varepsilon C_{n_{s} x m}, Z_{s} \varepsilon C_{n_{x} x n_{s}}, 1 \leq s \leq k$, such that

$$
H=\left[\begin{array}{ccc}
M_{11} & \cdot & \cdot M_{1 k} \\
\vdots & & \vdots \\
M_{n 1} & \cdot & \cdot M_{n k}
\end{array}\right] \operatorname{diag}\left(Z_{1}, \cdots, Z_{k}\right)\left[\begin{array}{ccc}
W_{11} & \cdot & \cdot W_{1 n} \\
\vdots & & \vdots \\
W_{k 1} & \cdot & \cdot W_{k n}
\end{array}\right],
$$

then the unique solution of (1.4) is given by

$$
X=\left\{\sum_{j=1}^{r} \sum_{h=1}^{j} \sum_{s=1}^{k} a_{j} M_{l s} Z_{s}^{h-1} W_{s n} S P^{j-h}\right\}\left\{\sum_{j=0}^{r} a_{j} P^{j}\right\}^{-1}
$$
PROOF. Note that $X$ is a solution of equation (1.4) if and only if $y=\left[\begin{array}{c}X \\ X P \\ \vdots \\ X P^{n-1}\end{array}\right]$ is a solution of
the equation 


$$
H Y-Y P=-\left[\begin{array}{c}
0 \\
\vdots \\
0 \\
S
\end{array}\right]
$$

From (2.2), equation (1.4) had only one solution, [12], [2], and from corollary 2 of [2], if $Y$ is the unique solution of (2.5), it follows that

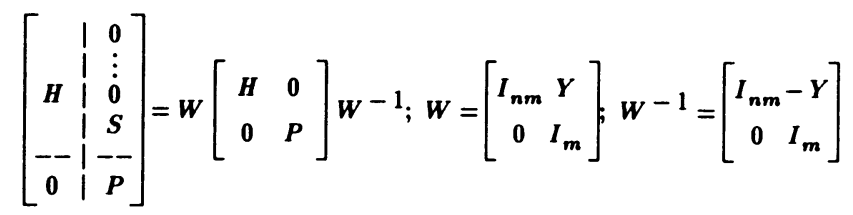

From (2.6) it follows that

$$
q(V)=W q\left(\left[\begin{array}{ll}
H & 0 \\
0 & P
\end{array}\right]\right) W^{-1}=W\left[\begin{array}{cc}
q(H) & 0 \\
0 & q(P)
\end{array}\right] W^{-1}=\left[\begin{array}{cc}
0 & Y q(P) \\
0 & q(P)
\end{array}\right]
$$

On the other hand, taking into account the block triangular structure of $V$ and the polynomial calculus it follows that

$$
q(V)=q\left(\left[\begin{array}{c|c}
H & 0 \\
& \vdots \\
\hdashline 0 & P
\end{array}\right]\right)=\left[\begin{array}{cc}
q(H) & N \\
0 & q(P)
\end{array}\right]
$$

for some matrix $N \varepsilon C_{m n x p}$. From the spectral mapping theorem ([3], p. 569), and (2.2) it follows that $q(P)$ is invertible. Hence from (2.7), (2.8), we have

$$
Y q(P)=N, \quad Y=N(q(P))^{-1}
$$

Considering the powers $V^{j}, 0 \leq j \leq r$, one gets that the $(i, 2)$ block entry of the operators $V^{j}$, denoted by $V_{i, 2}^{j}, 1 \leq i \leq 2,1 \leq j \leq r$, satisfy

$$
\begin{gathered}
V_{i, 2}^{j}=H V_{1,2}^{j-1}+\left[\begin{array}{c}
0 \\
\vdots \\
0 \\
S
\end{array}\right] v_{2,2}^{j-1} ; V_{2,2}^{j}=P^{j} \\
v_{1,2}^{0}=0, v_{2,2}^{0}=I_{m}
\end{gathered}
$$

By multiplying the matrix $V \dot{1,2}$ by the coefficients $a_{j}, 0 \leq j \leq r$, and by addition it follows that the entry $(1,2)$ of the block entry $q(V)$ takes the form

$$
N=\sum_{j=1}^{r} \sum_{h=1}^{j} a_{j} H^{h-1}\left[\begin{array}{c}
0 \\
\vdots \\
0 \\
S
\end{array}\right] P^{j-h}
$$

From (2.10), (2.3) and the relationship $X=[I, 0, \cdots, 0] Y, Y=N(q(P))^{-1}$, the result has been established.

Now we recall some definitions recently given in [7].

DEFINITION 1. Let $T_{j} \varepsilon \mathrm{C}_{m x m}$ for $0 \leq j \leq n-1$. We say that $(X, P)$ is a $(m, p)$ co-solution of the matrix equation

$$
Z^{n}+T_{n-1} Z^{n-1}+\cdots+T_{1} Z+T_{0}=0
$$

if $X \varepsilon \mathrm{C}_{m x p}, P \varepsilon \mathrm{C}_{p x p}, X \neq 0$ and

$$
X P^{n}+T_{n-1} X P^{n-1}+\cdots+T_{1} X P+T_{0} X=0
$$

Let $\left(X_{i}, P_{i}\right)$ be a $\left(m, n_{i}\right)$ co-solution of $(2.11)$ for $1 \leq i \leq k$. We say that the set $\left\{\left(X_{i}, P_{i}\right) ; 1 \leq i \leq k\right\}$ is 
a $k$-complete set of co-solutions of (2.11), if the block matrix $W=\left(W_{i, j}\right)$, with $W_{i j}=X_{j} P_{j}^{i-1}$, for $1 \leq i \leq n, 1 \leq j \leq k$, is invertible in $\mathrm{C}_{m n x m n}$.

The following result whose proof may be found in [7], provides $k$-complete sets of co-solutions of equation (2.11).

THEOREM 2. ([7]) Let $T \varepsilon C_{m x m}$ for $0 \leq i \leq n-1$, and let $H$ and $M$ be matrices given in Theorem 1 and satisfying (2.3). Then $\left\{\left(M_{1 s}, Z_{s}\right) ; 1 \leq s \leq k\right\}$ is a $k$-complete set of co-solutions of equation (2.11).

REMARK 1. Note that if $(X, P)$ is a $(m, p)$ co-solution of equation (2.11), then

$$
\left[\begin{array}{c}
X \\
X P \\
\vdots \\
X P^{n-1}
\end{array}\right] P=H\left[\begin{array}{c}
X \\
X P \\
\vdots \\
X P^{n}-1
\end{array}\right]
$$

where $H$ is the matrix defined by (2.1). Thus if $v$ is an eigenvector of $P$ corresponding to the eigenvalue $\lambda$, then

$$
H\left[\begin{array}{c}
X \\
X P \\
\vdots \\
X P^{n}-1
\end{array}\right] v=\left[\begin{array}{c}
X \\
X P \\
\vdots \\
X P^{n}-1
\end{array}\right] P v=\lambda\left[\begin{array}{c}
X \\
X P \\
\vdots \\
X P^{n}-1
\end{array}\right] v
$$

and if the rank of $X$ is $p \leq m$, it follows that

$$
\left[\begin{array}{c}
X \\
X P \\
\vdots \\
X P^{n-1}
\end{array}\right] v \neq 0, \quad \lambda \varepsilon \sigma(H) \text { and } \sigma(P) \subset \sigma(H)
$$

Furthermore, if the matrix $\boldsymbol{H}$ satisfies the spectral condition

$$
\text { If } z, w \varepsilon \sigma(H) \text { and } z \neq w \text {, then } z-w \text { is not an integer }
$$

then, for any positive integer $k \geq 1$, from (2.13)-(2.14) it follows that

$$
\sigma(k I+P) \cap \sigma(H)=\emptyset
$$

The following result is related to the concept of $k$-complete set of co-solutions for an equation of the type (2.11) and will be used in section 4.

LEMMA 1. Let $\left\{\left(X_{i}, P_{i}\right) ; 1 \leq j \leq k\right\}$ be a $k$-complete set of co-solutions of equation (2.11) with $X_{i} \varepsilon C_{m x n_{i}}, P_{i} \varepsilon C_{n_{i} x n_{i}}, 1 \leq i \leq k, n_{1}+n_{2}+\cdots+n_{k}=m n$. Then the block matrix

$$
S=\left[\begin{array}{cccc}
x_{1} & x_{2} & & x_{k} \\
x_{1} P_{1} & x_{2} P_{2} & & x_{k} P_{k} \\
X_{1} P_{1}\left(P_{1}-I\right) & x_{2} P_{2}\left(P_{2}-I\right) & & x_{k} P_{k}\left(P_{k}-I\right) \\
\vdots & \vdots & & \vdots \\
X_{1} \prod_{j=0}^{n} \bar{m}^{2}\left(P_{1}-j I\right) & x_{2} \prod_{j=0}^{n-2}\left(P_{2}-j I\right) & \cdots & x_{k} \prod_{j=0}^{n}-^{2}\left(P_{k}-j I\right)
\end{array}\right]
$$

is invertible in $\mathrm{C}_{m n x m n}$.

PROOF. Let $h$ be an integer such that $1 \leq h \leq n-2$ and let $B_{h}$ the block bi-diagonal matrix in $\mathbf{C}_{m n x m n}$ defined by

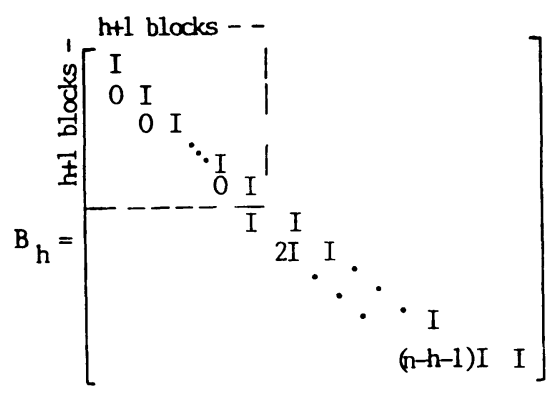


where $I$ denotes the identity matrix in $C_{m x m}$. If $W$ is the block Vandermonde matrix defined by

$$
W=\left[\begin{array}{cccccc}
X_{1} & x_{2} & \cdot & \cdot & \cdot & x_{k} \\
X_{1} P_{1} & x_{2} P_{2} & \cdot & \cdot & \cdot & x_{k} P_{k} \\
\vdots & & & & & \vdots \\
X_{1} P_{1}^{n-1} & x_{2} P_{2}^{n-1} & \cdot & \cdot & \cdot & x_{k} P_{k}^{n-1}
\end{array}\right]
$$

and associated to the $k$-complete set $\left\{\left(X_{i}, P_{i}\right) ; 1 \leq i \leq k\right\}$ of co-solutions of equation (2.11), then, straightforward computations show that

$$
W=B_{n-2} B_{n-3} \cdots B_{1} S
$$

Hence and the invertibility of $W$ and the matrices $B_{h}$ one concludes the invertibility of the matrix $S$. Thus the result is established.

\section{GENERAL RESULTS.}

We begin this section with a definition which generalizes the concept of a fundamental set of solutions given in [6] for second order matrix differential equations.

DEFINITION 2. Let $Q_{i}(t)$ for $0 \leq i \leq n-1, C_{m x m}$ valued continuous functions on an interval $J$ containing the origin of the real line, and let $Y_{i}(t)$ be a $C_{m x n_{i}}$ valued $n$-times continuously differentiable function in $J$ for $1 \leq i \leq k$. We say that the set $\left\{Y_{i} ; 1 \leq i \leq k\right\}$ is a $k$-fundamental set of solutions of equation (1.5), if any mxm solution $Y(t)$ of equation (1.5) in $J$, there exist matrices $R_{i} \varepsilon C_{n_{i} x m}$, uniquely determined by $Y(t)$, such that $n_{1}+n_{2}+\cdots+n_{k}=m n$ and

$$
Y(t)=Y_{1}(t) R_{1}+Y_{2}(t) R_{2}+\cdots+Y_{k}(t) R_{k}, \quad t \varepsilon J
$$

The following lemma provides an useful characterization of a $k$-fundamental set of solutions of equation (1.5).

LEMMA 2. Let $Y_{i}(t)$ be a $C_{m x n_{i}}$ valued solution of equation (1.5) defined in $J$ for $1 \leq i \leq k$ and $n_{1}+\cdots+n_{k}=m n$. Let $G(t)$ be the block matrix function defined by

$$
G(t)=\left[\begin{array}{cccc}
Y_{1}(t) & Y_{2}(t) & \cdots & Y_{k}(t) \\
Y_{1}^{(1)}(t) & Y_{2}^{(1)}(t) & \cdots & Y_{k}^{(1)}(t) \\
\vdots & & & \vdots \\
Y_{1}^{(n-1)}(t) & Y_{2}^{(n-1)}(t) & \cdots & Y_{k}^{(n-1)}(t)
\end{array}\right]
$$

Then $\left\{Y_{i} ; 1 \leq i \leq k\right\}$ is a $k$-fundamental set of solutions of (1.5) in $J$, if there exists a point $t_{1} \varepsilon J$ such that $G\left(t_{1}\right)$ is invertible in $\mathrm{C}_{m n x m n}$. In this case $G(t)$ is invertible for all $t$ in $J$.

PROOF. Since $Y_{i}(t)$ is a $C_{m x n_{i}}$ valued solution of (1.5) for $1 \leq i \leq k$, it is clear that $G(t)$ defined by (3.2) satisfies

$$
G^{(1)}(t)=\left[\begin{array}{ccccc}
0 & I & 0 & \cdots & 0 \\
0 & 0 & I & & 0 \\
\vdots & \vdots & \vdots & & \vdots \\
0 & 0 & & & I \\
-Q_{0}(t) & -Q_{1}(t) & & \cdots & -Q_{n-1}(t)
\end{array}\right] G(t) ; t \varepsilon J
$$

If $U(t, s)$ is the transition state matrix of system (3.3) such that $U(t, t)=I,([8]$, p. 598$)$, then we have $G(t)=U\left(t, t_{1}\right) G\left(t_{1}\right)$, for all $t \varepsilon J$. Given a solution $Y$ and its initial conditions $Y^{(h)}\left(t_{1}\right)=C_{h} \varepsilon \mathrm{C}_{m x m}$, for $O \leq h \leq n-1$, taking the matrices $R_{i} \varepsilon \mathrm{C}_{m x n_{i}}$ for $1 \leq i \leq k$, such that 


$$
\left[\begin{array}{c}
R_{1} \\
R_{2} \\
\vdots \\
R_{k}
\end{array}\right]=\left(G\left(t_{1}\right)\right)-1\left[\begin{array}{c}
C_{0} \\
C_{1} \\
\vdots \\
C_{n-1}
\end{array}\right]
$$

one determines uniquely the matrices $R_{i}$ such that satisfy (3.1) because the function $Y_{1}(t) R_{1}+\cdots+Y_{k}(t) R_{k}$ is a solution of (1.5) that satisfies the same initial conditions at $t_{1}$ as $Y(t)$. From the uniqueness for solutions of such a problem the result is concluded.

Now we consider the non-homogeneous problem

$$
y^{(n)}+Q_{n-1}(t) y^{(n-1)}+\cdots+Q_{0}(t) y=f(t), \quad t \varepsilon J
$$

where $f(t)$ is a continuous $C_{m x 1}$ valued function in the interval $J$.

Let us assume that $\left\{Y_{i} ; 1 \leq i \leq k\right\}$ is a $k$-fundamental set of solutions of the homogeneous equation (1.5), and let us look for solutions of the non-homogeneous equation (3.4) of the form

$$
y(t)=Y_{1}(t) R_{1}(t)+Y_{2}(t) R_{2}(t)+\cdots+Y_{k}(t) R_{k}(t),
$$

where $Y_{i}(t) \varepsilon \mathrm{C}_{m x n_{i}}$ and $R_{i}(t) \varepsilon \mathrm{C}_{n_{i} x 1}$ for $1 \leq i \leq k$. If we assume differentiability for the vector functions $R_{i}$ and we impose that $R_{i}$ satisfy

$$
G(t)\left[\begin{array}{c}
R_{1}^{(1)}(t) \\
\vdots \\
R_{k}^{(1)}(t)
\end{array}\right]=\left[\begin{array}{c}
0 \\
\vdots \\
0 \\
f(t)
\end{array}\right]
$$

From (3.6), the derivatives of $y(t)$ defined by (3.5) take the form

Hence it follows that

$$
\begin{gathered}
y^{(h)}(t)=\sum_{i=1}^{k} Y_{i}^{(h)}(t) R_{i}(t), \quad 1 \leq h \leq n-1, \\
y^{(n)}(t)=\sum_{i=1}^{k} Y_{i}^{(n)}(t) R_{i}(t)+f(t)
\end{gathered}
$$

$$
\begin{gathered}
y^{(n)}(t)+Q_{n-1}(t) y^{(n-1)}(t)+\cdots+Q_{0}(t) y(t)= \\
\sum_{i=1}^{k}\left\{y_{i}^{(n)}(t)+Q_{n-1}(t) Y_{i}^{(n-1)}(t)+\cdots+Q_{0}(t) Y_{i}(t)\right\} R_{i}(t)+f(t)=f(t)
\end{gathered}
$$

because each $Y_{i}(t)$ is a $C_{m x n_{i}}$ solution of the homogeneous equation (1.5), for $1 \leq i \leq k$. Since $\left\{Y_{i} ; 1 \leq j \leq k\right\}$ is a $k$-fundamental set of solutions of $(1.5)$, the matrix $G(t)$ is invertible. Let $T(t)=\left(T_{i j}(t)\right)=(G(t))^{-1}$, with $T_{i j}(t) \varepsilon C_{n_{i} x m}$, for $1 \leq j \leq n, 1 \leq i \leq k$. Then by integration of (3.6) it follows that

$$
R_{i}(t)=\int_{t_{1}}^{t} T_{i n}(s) f(s) d s+D_{i} ; \quad D_{i} \varepsilon C_{n_{i} x 1}, \quad 1 \leq i \leq k, \quad t_{1}^{\varepsilon J}
$$

Taking $D_{i}=0$, for $1 \leq i \leq k$, a particular solution $y_{p}(t)$ of (3.4) satisfying $Y_{p}^{(h)}\left(t_{1}\right)=0,0 \leq h \leq n-1$, is given by

$$
y_{p}(t)=\sum_{i=1}^{k} Y_{i}(t) R_{i}(t)=\int_{t_{1}}^{t}\left\{\sum_{i=1}^{k} Y_{i}(t) T_{i n}(s) f(s)\right\} d s
$$

From the previous comments and Lemma 2, the following result has been proved:

THEOREM 3. Let $\left\{Y_{i} ; 1 \leq i \leq k\right\}$ be a $k$ fundamental set of solutions of equation (1.5) in $J$, where $Y_{i}(t) \varepsilon \mathbf{C}_{m x n_{i}}$ and $n_{1}+\cdots+n_{k}=n m$. If $f(t)$ is a continuous function in $J$ and 
$T(t)=\left(T_{i j}(t)\right)=(G(t))^{-1}$, with $T_{i j}(t) \varepsilon C_{n_{i} x n}$, for $1 \leq i \leq k, \quad 1 \leq j \leq n$, and $G(t)$ is defined by (3.2), then the general solution of (3.4) is defined by (3.5) and $R_{i}(t)$ takes the form (3.9) for $1 \leq i \leq k$.

\section{MATRIX SERIES SOLUTIONS: CONSTRUCTION, CONVERGENCE AND \\ APPLICATIONS.}

Let us suppose that $P_{j}(t)$ is an analytic matrix function in $|t|<a$, for $0 \leq j \leq n-1$, and let us consider the power expansion

$$
P_{h}(t)=\sum_{j \geq 0} P_{h, j} t^{j}, \quad|t|<a, \quad P_{h, j} \varepsilon \mathbf{C}_{m x m}, \quad 0 \leq h \leq n-1, j \geq 0
$$

Let $Z \varepsilon C_{p x p}$ with $p \leq m$, and let us look for solutions of equation (1.1) of the type

$$
X(t)=\left(\sum_{j \geq 0} C_{j} t^{j}\right) t^{Z}, 0<t<a, C_{j} \varepsilon \mathbf{C}_{m x p},
$$

where $t^{Z}=\exp (Z 1 n(t))$. Taking formal derivatives of $X(t)$ given by (4.2) and substituting into (1.1) one gets

$$
\begin{gathered}
t^{n} \sum_{j \geq 0} C_{j} \prod_{s=0}^{n-1}(Z+(j-s) I) t^{Z+(j-n) I}+t^{n-1}\left\{\sum_{j \geq 0} P_{n-1, j} t^{j}\right\} \\
\left\{\sum_{j \geq 0} C_{j} \prod_{s=0}^{n-2}(Z+(j-s) I) t^{Z+(j-n-1) I}\right\}+\cdots+t \\
\left\{\sum_{j \geq 0} P_{1, j} t^{j}\right\}\left\{\sum_{j \geq 0} C_{j}(Z+j I) t^{Z+(j-1) I}\right\}+\left\{\sum_{j \geq 0} P_{0, j} t^{j}\right\}\left\{\sum_{j \geq 0} C_{j} t^{Z+j I}\right\}=0 \\
\left\{\sum _ { j \geq 0 } \left[C_{j}^{n} \prod_{s=0}^{1}(Z+(j-s) I)+\sum_{q=0}^{j} P_{n-1, j-q} C_{q} \prod_{s=0}^{2}(Z+(q-s) I)+\cdots\right.\right. \\
\left.\left.+\sum_{q=0}^{j} P_{1, j-q} C_{q}(Z+q I)+\sum_{q=0}^{j} P_{0, j-q} C_{q}\right] t^{j}\right\} t^{Z=0}
\end{gathered}
$$

that may be written in the form

$$
\left[\sum_{j \geq 0}\left\{C_{j} \prod_{s=0}^{n-1}(Z+(j-s) I)+\sum_{r=0}^{n-1} \sum_{q=0}^{j} P_{r, j-q} C_{q} \prod_{s=0}^{r-1}(Z+(q-s) I)+\sum_{q=0}^{j} P_{0, j-q} C_{q}\right\} t^{j}\right] t^{Z}=0
$$

Equating to the zero matrix the coefficient of $t^{0}$ appearing in (4.4), it follows that coefficients $C_{j}$ must satisfy

$$
C_{0} \prod_{s=0}^{n-1}(Z-s I)+\sum_{r=1}^{n-1} P_{r, 0} C_{0} \prod_{s=0}^{r-1}(Z-s I)+P_{0,0} C_{0}=0
$$

Now we are interested in writing equation (4.5) in the form of equation of the type (1.4) for $C_{0}$. If we denote by $M_{n}(Z)-\prod_{s=0}^{n}(Z=s I)$, then equation (4.5) takes' the form

$$
C_{0} M_{n-1}(Z)+\sum_{r=1}^{n-1} P_{r, 0} C_{0} M_{r-1}(Z)+P_{0,0} C_{0}=0
$$

If we equate to the zero matrix the coefficient of $t^{j}$ for $j \geq 1$ in (4.4), we have

where

$$
C_{j} M_{n-1}(Z+j I)+\sum_{r=1}^{n-1} P_{r, 0} C_{j} M_{r-1}(Z+j I)+P_{0,0} C_{j}=D_{j}, j \geq 1
$$

$$
A_{q}=\sum_{r=1}^{n-1} P_{r, j-q} C_{q} M_{r-1}(Z+q I)+P_{0, j-q} C_{q} \text { and } D_{j}=-\sum_{q=0}^{j-1} A_{q}
$$


depends on the previous coefficients $C_{0}, C_{1}, \cdots, C_{j-1}$.

Easy computations show that

and

$$
\left.M_{n}(Z)=\prod_{s=0}^{n}(Z-s I)=\prod_{s=1}^{n}(Z-s I)\right) Z=P_{n}(Z) Z, P_{n}(Z)=\prod_{s=1}^{n}(Z-s I)
$$

$$
P_{n}(Z)=Z^{n}+A_{n-1} Z^{n-1}+\cdots+A_{1} Z+A_{0}
$$

where

$$
\begin{gathered}
A_{c}=(-1)^{n-c} S_{n, n-c}, S_{n, n-c}=_{1 \leq i_{1}<\cdots<i_{n-c} \leq n} \sum_{i_{1} i_{2} \cdots i_{n-c} I, 1 \leq c \leq n-1,} \\
S_{n, n}=n ! I, S_{n, 0}=I
\end{gathered}
$$

and the sequence $S_{n, c}$ is related by the recurrence relationship

$$
S_{n, 0}=I, S_{n, c}=n S_{n-1, c-1}+(n-1) S_{n-2, c-1}+\cdots+c S_{c-1, c-1} \text {, for } 1 \leq c \leq n
$$

Hence equation (4.6) for $C_{0}$ may be written as an algebraic matrix equation of polynomial type of the form

$$
\begin{gathered}
T_{0}=P_{0,0} Z^{n}+\sum_{j-1}^{n-1} T_{j} C_{0} Z^{j}+T_{0} C_{0}=0 ; \\
T_{j}=\sum_{r=j}^{n} P_{r, 0} B(r, j), B(r, j)=(-1)^{r-j} S_{r-1, r-j}, \quad 1 \leq j \leq n-1
\end{gathered}
$$

while (4.7) may be written in the form

with

$$
C_{j}(Z+j I)^{n}+\sum_{q=1}^{n-1} T_{q} C_{j}(Z+j I)^{q}+P_{0,0} C_{j}=D_{j}
$$

$$
A_{q}=\sum_{i=1}^{n-1} T_{*}(j-q, i) C_{q}(Z+q I)^{i}+P_{0, j-q} C_{q}, T_{*}(j-q, i)=\sum_{r=i}^{n-1} P_{r, j-q} B(r, i) D_{j}=-\sum_{q=0}^{j-1} A_{q}
$$

Note that from Definition 1, equation (4.9) means that $\left(C_{0}, Z\right)$ is a $(m, p)$ co-solution of equation

$$
V^{n}+T_{n-1} V^{n-1}+\cdots+T_{1} V+T_{0}=0
$$

where $T_{j} \varepsilon C_{m x m}$ is defined by (4.10), for $0 \leq j \leq n-1$. If we take $P=Z+j I \varepsilon C_{p x p}$, and we assume that the matrix $H$ defined by (2.1) satisfies the condition (2.14), $q(z)=\sum_{j=0}^{r} a_{j} z^{j}$ is an annihilating polynomial of $H$, and $M=\left(M_{i j}\right), W=\left(W_{s i}\right)=M^{-1}$, and $D=\operatorname{diag}\left(Z_{1}, \cdots, Z_{k}\right)$, satisfy the condition (2.3), then, from Theorem 1 , the unique solution $C_{j}$ of the matrix equation (4.11) is given by

$$
C_{j}=\left\{\sum_{v=1}^{r} \sum_{h=1}^{v} \sum_{s=1}^{k} a_{v} M_{1 s} Z_{s}^{h-1} W_{s 2} D_{j}(Z+j I)^{v-h}\right\}\left\{\sum_{v=0}^{r} a_{v}(Z+j I)^{v}\right\}^{-1}, j \geq 1
$$

Note that from Theorem 2, a $k$-complete set of co-solutions of the algebraic matrix equation (4.13), is defined by $\left\{\left(M_{1 s}, Z_{s}\right) ; 1 \leq s \leq k\right\}$, where $M_{1 s}$ and $Z_{s}$ are given by (2.3). Thus we may take as $Z$ as each of the matrices $Z_{s}$ and $C_{0}(s)=M_{1 s}$, for $1 \leq s \leq k$, in the series solution given in (4.2), we obtain

and

$$
X(t, s)=U(t, s) t^{Z} s=\left(\sum_{j \geq 0} C_{j}(s) t^{j}\right) t^{Z} s, \quad 0<t<a, \quad 1 \leq s \leq k
$$




$$
\begin{gathered}
C_{j}(s)=\left\{\sum_{v=1}^{r} \sum_{h=1}^{v} \sum_{w=1}^{k} a_{v} M_{1} z_{w}^{h-1} W_{w n} D_{j}(s)\left(Z_{s}+j I\right)^{v-h}\right\}\left\{\sum_{v=0}^{r} a_{v}\left(Z_{s}+j I\right)^{v}\right\}-1, \\
A_{q}(s)=\sum_{i=1}^{n-1} T_{*}(j-q, i) C_{q}(s)\left(Z_{s}+q I\right)^{i}+P_{0, j-q} C_{q}(s), D_{j}(s)=-\sum_{q=0}^{j-1} A_{q}(s), 1 \leq s \leq k
\end{gathered}
$$

where $T_{*}(j-q, i)$ is defined in (4.12).

Now we prove that if $\left(C_{0}, Z\right)$ is a $(m, p)$ co-solution of $(4.13)$ and $C_{j}$ is given by $(4.14)$, for $j \geq 1$, then $X(t)$ defined by (4.2) is a $C_{m x p}$ valued solution of (1.1). From the analyticity of $P_{h}(t)$, for $0 \leq h \leq n-1$, the coefficients $P_{h, j}$ of (4.1) satisfy the inequalities

$$
\left\|P_{h, j}\right\| \rho^{j} \leq L, \text { for } 0<\rho<a, \quad 0 \leq h \leq n-1, \quad j \geq 0
$$

Let $M$ be an upper bound of $\left\|T_{j}\right\|$ for $0 \leq j \leq n-1$, then from the definition of $T_{*}$ we have

$$
\left\|T_{*}(j-q, i)\right\| \leq N \rho^{q-j}, \quad N=n M, \quad 0 \leq q \leq j-1, \quad 1 \leq i \leq n-1
$$

Taking norms in (4.11) it follows that

$$
\begin{aligned}
\left\|D_{j}\right\| & \geq\left\|C_{j}(Z+j I)^{n}\right\|-\left\|\sum_{q=1}^{n-1} T_{q} C_{j}(Z+j I)^{q}\right\|-\left\|P_{0,0} C_{j}\right\| \geq \\
& \geq\left\|C_{j}\left(j^{n} I+\sum_{q=1}^{n}\left(\begin{array}{l}
n \\
q
\end{array}\right) j^{n-q} Z^{q}\right)\right\|-\sum_{q=1}^{n-1}\left\|T_{j}\right\|\left\|C_{q}\right\|\|Z+j I\|^{q}-\left\|P_{0,0}\right\|\left\|C_{j}\right\| \\
& \geq\left\{j^{n}-\sum_{q=1}^{n}\left(\begin{array}{l}
n \\
q
\end{array}\right) j^{n-q}\|Z\|^{q}-\sum_{q=1}^{n-1} N\|Z+j I\|^{q}-\left\|P_{0,0}\right\|\right\}\left\|C_{j}\right\|
\end{aligned}
$$

From (4.12) and (4.19) it follows that

$$
\begin{aligned}
\left\|D_{j}\right\| & \leq \sum_{q=0}^{j-1} \sum_{i=1}^{n-1}\left\|T_{*}(j-q, i)\right\|\left\|C_{q}\right\|\|Z+q I\|^{i}+\left\|P_{0, j-q}\right\|\left\|C_{q}\right\| \\
& \leq \sum_{q=0}^{j-1}\left\{\sum_{i=1}^{n-1} \rho^{q-j}\left|N\|Z+q I\|^{i}\right|+L\right\}\left\|C_{q}\right\| \\
& \leq \sum_{q=0}^{j-1} \rho^{q-1}\left(n N(\|Z\|+q)^{n-1}+L\right)\left\|C_{q}\right\|
\end{aligned}
$$

Let $j_{0}$ be the first positive integer $j$ such that

$$
j^{n}-\sum_{q=0}^{n}\left(\begin{array}{l}
n \\
q
\end{array}\right) j^{n-q}\|Z\|^{q}-\sum_{q=1}^{n-1} N\|Z+j I\|^{q}-\left\|P_{0,0}\right\|>0
$$

and let us define by $\left\{\gamma_{j}\right\}$ the sequence of positive scalars such that

$$
\gamma_{j}=\left\|C_{j}\right\|, j=0,1,2, \cdots, j_{0}-1
$$

and for $j \geq j_{0}$ let $\gamma_{j}$ be defined by the equation

$$
\begin{gathered}
\left\{j^{n}-\left(\sum_{q=1}^{n}\left(\begin{array}{l}
n \\
q
\end{array}\right) j^{n-q}\|Z\|^{q}+N \sum_{q=1}^{n-1}\|Z+j I\|^{q}+\left\|P_{0,0}\right\|\right)\right\} \gamma_{j} \\
=\sum_{q=0}^{j-1} \rho^{q-j}\left(n N(\|Z\|+q)^{n-1}+L \mid \gamma_{q}\right.
\end{gathered}
$$

From the definition of $\gamma_{j}$ it follows that

$$
\left\|C_{j}\right\| \leq \gamma_{j}, \quad j \geq 0
$$


On the other hand, from the definition of $\gamma_{j}$ it follows that

$$
\begin{aligned}
& \frac{\gamma_{j+1}|t|^{j+1}}{\gamma_{j}|t|^{j}}=|t| \frac{\sum_{q=0}^{j-1} \rho^{q-j-1}\left(n N(\|Z\|+q)^{n-1}+L\right) \gamma_{q}+\rho^{-1}\left(n N(\|Z\|+j)^{n-1}+L\right) \gamma_{j}}{\left\{(j+1)^{n}-\left(\sum_{q=1}^{n}\left(\begin{array}{l}
n \\
q
\end{array}\right)(j+1)^{n-q}\|Z\|^{q}+N \sum_{q=1}^{n-1}\|Z+(j+1) I\|^{q}+\left\|P_{0,0}\right\|\right\}_{j}\right.} \\
= & \frac{|t|}{\rho} \frac{\left\{j^{n}-\left(\sum_{q=1}^{n}\left(\begin{array}{l}
n \\
q
\end{array}\right) j^{n-q}\|Z\|^{q}+N \sum_{q=1}^{n-1}\|Z+j I\|^{q}+\left\|P_{0,0}\right\|\right)+n N(\|Z\|+j)^{n-1}+L\right\} \gamma_{j}}{\left\{(j+1)^{n}-\left(\sum_{q=1}^{n}\left(\begin{array}{l}
n \\
q
\end{array}\right)(j+1)^{n-q}\|Z\|^{q}+N \sum_{q=1}^{n-1}\|Z+(j+1) I\|^{q}+\left\|P_{0,0}\right\| \gamma_{j}\right.\right.}
\end{aligned}
$$

Taking limits as $j \rightarrow \infty$ in (4.26) we obtain that this limit is $|t| / \rho$ and thus the series

$$
U(t, Z)=\sum_{j \geq 0}\left\|C_{j} t^{j}\right\| \text { is convergent for }|t|<\rho<a
$$

Let us consider $\left\{\left(M_{1 s}, Z_{s}\right) ; 1 \leq s \leq k\right\}$ be the $k$-complete set of co-solutions of the algebraic matrix equation (4.13) where $T_{j}$ for $0 \leq j \leq n-1$, are given by (4.10). Let us construct the set of series solution of equation (1.1) of the form

$$
X(t, s)=U(t, s) t^{Z} s=\left(\sum_{j \geq 0} C_{j}(s) t^{j}\right) z^{Z}, C_{0}(s)=M_{1 s}, 0<t<a, 1 \leq s \leq k,
$$

where $M=\left(M_{i j}\right)$ and $\left(Z_{1}, \cdots, Z_{k}\right)$ satisfy $(2.3)$ with $W=\left(W_{s i}\right)=M^{-1}$. Now we prove that the set $\{X(, s) ; 1 \leq s \leq k\}$ is a $k$-fundamental set of solutions of equation $(1.1)$ in the interval $J=(0, a)$. From Lemma 2, it is sufficient to prove that the block matrix function $G(t)$ given by

$$
\begin{aligned}
& G(t)=\left(G_{i s}(t)\right), G_{i s}(t)=X^{(i-1)}(t, s) ; 1 \leq i \leq n, 1 \leq s \leq k, G_{i s}(t) \varepsilon \mathbf{C}_{m x n_{i}} \\
& n_{1}+\cdots+n_{k}=m n, 0<i<a,
\end{aligned}
$$

is invertible in $\mathbf{C}_{m n x m n}$ for some $t_{1} \varepsilon(0, a)$.

Direct computations show that $G(t)=\left(G_{i s}(t)\right)$ defined by (4.28) may be written in the form

where

$$
G(t)=\operatorname{diag}\left(I, t^{-1} I, \cdots, t^{-n+1} I\right)\left(H_{i s}(t)\right) \operatorname{diag}\left(t^{Z}, \ldots, t^{Z}\right)
$$

$$
\begin{gathered}
H_{1 s}(t)=U(t, s) \\
H_{2 s}(t)=U^{(1)}(t, s) t+U(t, s) Z_{s} \\
H_{3 s}(t)=U^{(2)}(t, s) t^{2}+2 U^{(1)}(t, s) Z_{s} t+U(t, s) Z_{s}\left(Z_{s}-I\right)
\end{gathered}
$$$$
\boldsymbol{H}_{n s}(t)=U^{(n-1)}(t, s) t^{n-1}+\left(\begin{array}{c}
n-1 \\
1
\end{array}\right) U^{(n-2)}(t, s) Z_{s} t^{n-2}+\cdots+\left(\begin{array}{c}
n-1 \\
n-1
\end{array}\right) U(t, s) Z_{s}\left(Z_{s}-I\right) \cdots\left(Z_{s}-(n-2) I\right)
$$

Note that from (4.29), $G(t)$ is invertible at $t \varepsilon(0, a)$, if and only if $H(t)=\left(H_{i s}(t)\right)$ is invertible at $t$. On the other hand, from (4.30) it follows that

$$
H(0)=\left[\begin{array}{cccc}
C_{0}(1) & C_{0}(2) & \cdots & C_{0}(k) \\
C_{0}(1) Z_{1} & C_{0}(2) Z_{2} & \cdots & C_{0}(k) Z_{k} \\
C_{0}(1) Z_{1}\left(Z_{1}-I\right) & C_{0}(2) Z_{2}\left(Z_{2}-I\right) & \cdots & C_{0}(k) Z_{k}\left(Z_{k}-I\right) \\
\vdots & \vdots & & \vdots \\
C_{0}(1) \prod_{j=0}^{n}-^{2}\left(Z_{1}-j I\right) & C_{0}(2) \prod_{j=0}^{n}-^{2}\left(Z_{2}-j I\right) & \cdots & C_{0}(k) \prod_{j=0}^{n}-^{2}\left(Z_{k}-j I\right)
\end{array}\right]
$$


Since $\left\{\left(C_{0}(s), Z_{s}\right) ; 1 \leq s \leq k\right\}$ is a $k$-complete set of solutions of equation (4.13) from Lemma 1 , it follows that the matrix $H(0)$ is invertible in $C_{m n x m n}$. Note that $H(t)=\left(H_{i s}(t)\right)$ defined by $(4.30)$ is an analytic function in $|t|<a$ and from the invertibility of $H(0)$ and the perturbation lemma, ([10], p 32), there exists a positive number $b$ with $0<b \leq a$, such that $H(t)$ is invertible in $(0, b)$. Taking $t_{1}$ in $(0, b)$, it follows the invertibility of $H\left(t_{1}\right)$ and $G\left(t_{1}\right)$. Hence the following result has been established:

THEOREM 4. Let $P_{h}(t)$ be an analytic $C_{m x m}$ valued function in $|t|<a$ whose power series expansion is given by (4.1) for $0 \leq h \leq n-1$, and let $H$ be the matrix defined by (2.1) where $T_{h}$ is defined by (4.10) for $0 \leq h \leq n-1$. Let $q(z)={ }_{v}^{r}{ }_{0}^{r} a_{v} z^{v}$ be an annihilating polynomial of $H$ and let $M=\left(M_{i, j}\right), W=\left(W_{s i}\right)=M^{-1}$ and $Z_{1}, Z_{2}, \cdots, z_{k}$ be matrices satisfying (2.3) and let $X(t, s)$ be the generalized matrix power series of the form (4.15)-(4.17) constructed in terms of a $k$-complete set of co-solutions $\left\{\left(C_{0}(s), Z_{s}\right), 1 \leq s \leq k\right\}$ of the algebraic matrix equation (4.13). Then $\{X(\cdot, s) ; 1 \leq s \leq k\}$ is a $k$-fundamental set of solutions of equation $(1.1)$ in the interval $(0, a)$.

Now let us consider the non-homogeneous system (1.3) where $g(t)$ is a continuous function in $(0, a)$. Let $V(t)=(H(t))^{-1}$ where $H(t)$ is defined by (4.30) in terms of the $k$-fundamental set of solutions of (1.1) given in Theorem 4. Let us consider a block partition of $V(t)$ of the form

$$
V(t)=\left(V_{i j}(t)\right), \quad V_{i j}(t) \varepsilon \mathrm{C}_{n_{i} x m}, \quad 1 \leq i \leq k, \quad 1 \leq j \leq n
$$

Then from Theorems 3 and 4 , the general solution of $(1.3)$ in $(0, a)$ is given by

$$
x(t)=\sum_{i=1}^{k} X(t, i) D_{i}+\sum_{i=1}^{k} X(t, i) \int_{t_{1}}^{t} u^{-Z_{i}} V_{i n}(u)(g(u) / u) d u, D_{i} \varepsilon C_{n_{i} x 1},
$$

where we have taken into account the following expression for the inverse of $G(t)$ defined by (4.28):

$$
(G(t))^{-1}=\operatorname{diag}\left(t^{-Z_{1}}, \ldots, t^{-Z_{k}}\right)(H(t))^{-1} \operatorname{diag}\left(I, t I, \cdots, t^{n-1} I\right)
$$

and that system (1.3) may be written in the form

$$
x^{(n)}+t^{-1} P_{n-1}(t)+\cdots+t^{-n} P_{0}(t)=t^{-n} g(t),
$$

Since $X(t, i)=U(t, i) t Z_{i}$, expression (4.33) may be written in the form

$$
x(t)=\sum_{i=1}^{k} U(t, i) t Z^{Z}\left\{D_{i}+\int_{t_{1}}^{t} u^{-Z_{i}} V_{i n}(u)(g(u) / u) d u\right\}
$$

and from the comments previous to (3.10), a particular solution of (1.3) which satisfies $y^{(h)}\left(t_{1}\right)=0$, for $0 \leq h \leq n-1$, is given by

$$
y_{p}(t)=\sum_{i=1}^{k} U(t, i) \int_{t_{1}}^{t}(t / u) Z_{i V_{i n}(u)(g(u) / u) d u}
$$

In order to construct the solution of (1.3) which satisfies the initial conditions

$$
x\left(t_{1}\right)=c_{0}, x^{(1)}\left(t_{1}\right)=C_{1}, \cdots x^{(n-1)}\left(t_{1}\right)=c_{n-1}
$$

where $c_{h} \varepsilon \mathrm{C}_{m x 1}$ for $0 \leq h \leq n-1$, it is sufficient to take in (4.35), constants $D_{i} \varepsilon \mathbf{C}_{n_{i} x 1}, 1 \leq i \leq k$, such that

$$
G\left(t_{1}\right)\left[\begin{array}{c}
D_{1} \\
D_{2} \\
\vdots \\
D_{k}
\end{array}\right]=\left[\begin{array}{c}
c_{0} \\
\vdots \\
c_{n-1}
\end{array}\right]
$$


From (4.34) and (4.32), it follows that

$$
D_{i}=\left(t_{1}\right)^{-Z_{i}} \sum_{j=1}^{n} V_{i j}\left(t_{1}\right)\left(t_{1}\right)^{j-1} I_{m^{c}} c_{-1}, \quad 1 \leq i \leq k
$$

where $I_{m}$ denotes the identity matrix in $C_{m x m}$. Summarizing the following result has been established:

THEOREM 5. Let us consider the hypotheses and the notation of Theorem 4, where $g(t)$ is a continuous function in the interval $(0, a)$. Then the following results hold:

(i) The general solution of (1.3) is given by (4.35), where $D_{i} \varepsilon C_{n_{i} x 1}$ is an arbitrary vector for $1 \leq i \leq k$.

(ii) A particular solution of (1.3) satisfying $x^{(h)}\left(t_{1}\right)=0$ for $0 \leq h \leq n-1$, is given by (4.36). The unique solution which satisfies the initial condition (4.37) is given by (4.35), where $D_{i}$ is determined by (4.39) for $1 \leq i \leq k$.

ACKNOWLEDGEMENT. This work has been partially supported by the D.G.I.C.Y.T. grant PS90-0140.

\section{REFERENCES}

1. CODDINGTON, E.A. \& LEVINSON, N., Theory of Ordinary Differential Equations, McGraw-Hill, New York, 1955.

2. DAVIS, C. \& ROSENTHAL, P., Solving linear operator equations, Can. J. Math. XXVI 6 (1974), 1384-1389.

3. DUNFORD, N. \& SCHWARTZ, J., Linear Operators, Part I, Interscience, New York, 1957.

4. HILLE, E., Lectures on Ordinary Differential Equations, Addison Wesley Pubs. Co., 1969.

5. INCE, E.L., Ordinary Differential Equations, Dover Pubs. Co., New York, 1927.

6. JÓDAR, L., Explicit solutions for second order operator differential equations with two boundary value conditions, Linear Algebra Appl. 103 (1988), 73-86.

7. JÓDAR, L. \& NAVARRO, E., Rectangular co-solutions of polynomial matrix equations and applications, Appl. Maths. Letters 4 (1991), 13-16.

8. KAILATH, T., Linear Systems, Prentice Hall, Inc., Englewood Cliffs, New Jersey, 1980.

9. KELLER, H.B. \& WOLFE, A.W., On the nonunique equilibrium states and buckling mechanism of spherical shells, J. Soc. Indust. Appl. Math., 13 (1965), 674-705.

10. ORTEGA, J.M., Numerical Analysis, A Second Course, Academic Press, New York, 1972.

11. PARTER, S.V.; STEIN, M.L. \& STEIN, P.R., On the multiplicity of solutions of a differential equation arising in chemical reactor theory, Tech. Rep. 19/, Department of Computer Science, University of Wisconsin, Madison, 1973.

12. ROSENBLUM, M., On the operator equation BX-XA=Q, Duk. Math. J. 23 (1956), 263-269.

13. WEINMULLER, E., A difference method for a singular boundary value problem of second order, Math. and Computation 42, No. 166 (1984), 441-464. 


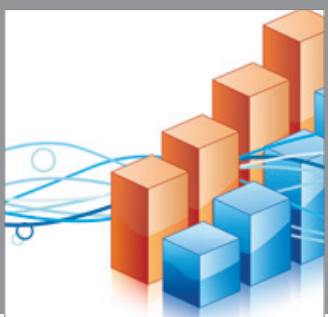

Advances in

Operations Research

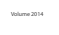

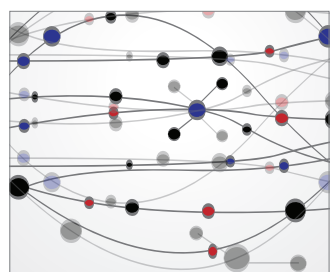

\section{The Scientific} World Journal
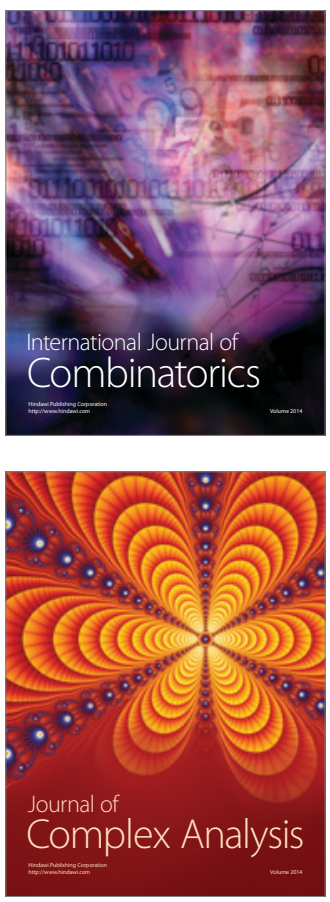

International Journal of

Mathematics and

Mathematical

Sciences
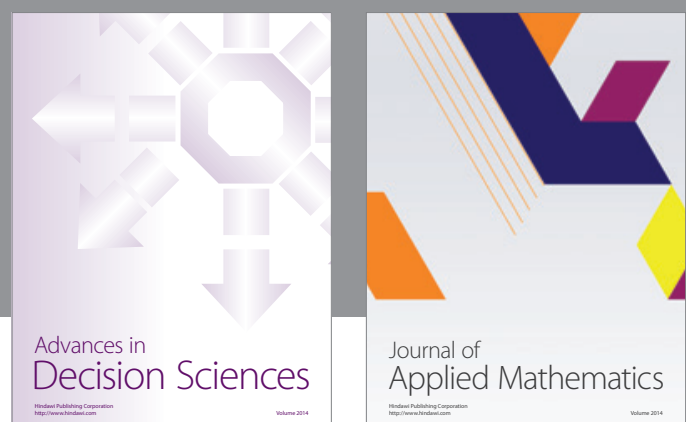

Journal of

Applied Mathematics
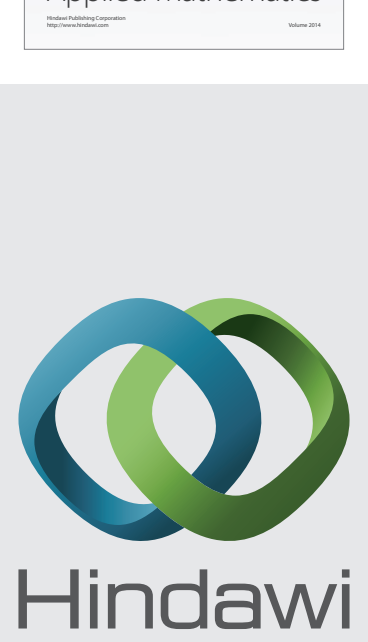

Submit your manuscripts at http://www.hindawi.com
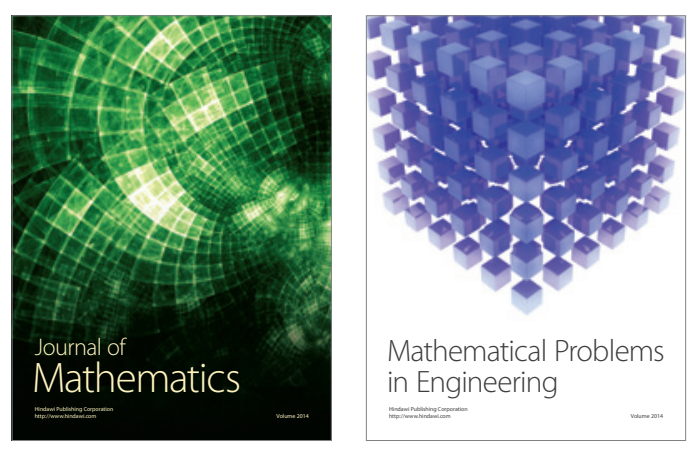

Mathematical Problems in Engineering
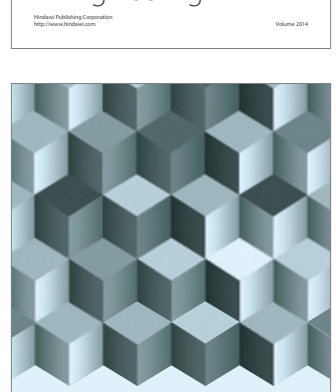

Journal of

Function Spaces
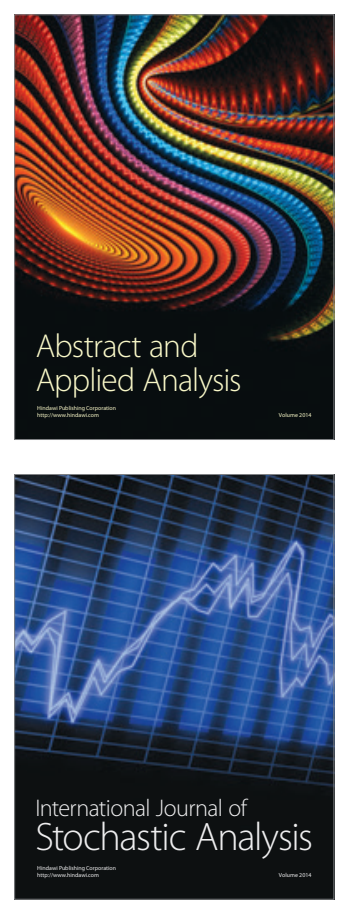

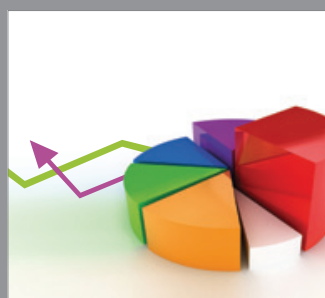

ournal of

Probability and Statistics

Promensencen
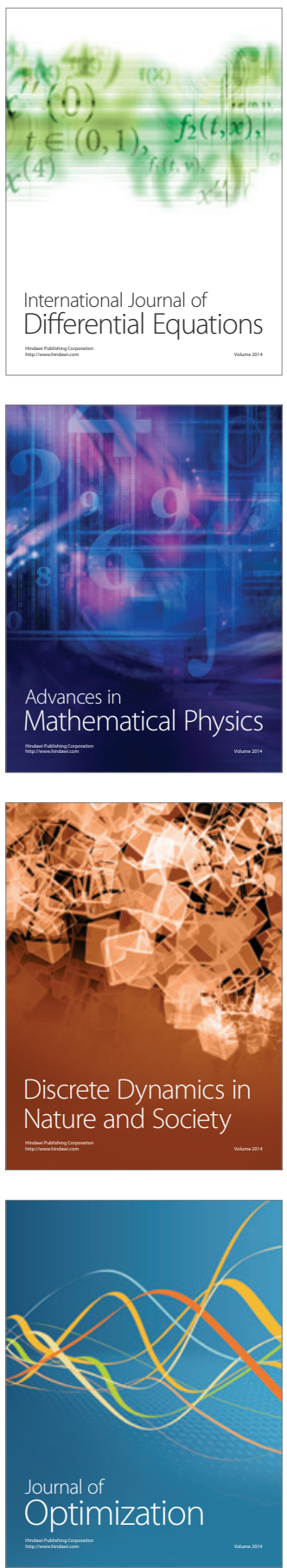